\title{
Effect of cardiac resynchronisation therapy on occurrence of ventricular arrhythmia in patients with implantable cardioverter defibrillators undergoing upgrade to cardiac resynchronisation therapy devices
}

\author{
G Lin, R F Rea, S C Hammill, D L Hayes, P A Brady
}

Division of Cardiovascular Diseases, Mayo Clinic, Rochester, MN, USA

Correspondence to: Dr Peter A Brady, MD, FRCP Mayo Clinic, 200 First Street SW, Rochester, MN, 55905, USA; brady.peter@mayo.edu

Accepted 14 August 2007 Published Online First 29 August 2007

\begin{abstract}
Background: Cardiac resynchronisation therapy (CRT) improves outcomes in selected patients with heart failure and left ventricular dysfunction. One mechanism of benefit is believed to be favourable ventricular remodelling. Whether CRT also decreases the frequency of ventricular arrhythmias and risk of sudden death is unknown.
\end{abstract}

Objective: To determine the effect of CRT on frequency of ventricular arrhythmias and appropriate ICD therapies.

Design: Retrospective cohort study.

Setting: Single-centre, tertiary care facility (Mayo Clinic). Patients: 52 patients (46 male), aged 70 (SD 10) years, who underwent upgrade from an implantable cardioverter defibrillator (ICD) to a CRT-defibrillator were included.

Interventions: Upgrade of ICD to CRT-defibrillator. Main Outcome Measures: Frequency of ventricular arrhythmias prior to and following upgrade to CRT device. Results: Ejection fraction increased from 22\% (SD 8\%) to $27 \%$ (SD 11\%) following CRT. However, the frequency of non-sustained ventricular arrhythmias, sustained ventricular arrhythmias, and ventricular fibrillation was not significantly changed prior to and following CRT (2.38 (SD 9.78) vs 58.51 (SD 412.73) per patient per month, $p=0.66 ; 0.07$ (SD 0.17) vs 0.16 (SD 0.52), $p=0.70$; 0.05 (SD 0.12) vs 0.25 (SD 1.40), $p=0.12$ ).

Conclusions: CRT is not associated with a decrease in the frequency of ventricular arrhythmia or appropriate device therapy. Thus, use of CRT alone is not beneficial in decreasing the frequency of ventricular arrhythmias or the risk of appropriate ICD therapies.

Cardiac resynchronisation therapy (CRT) is associated with fewer hospitalisations and decreased mortality in patients with congestive heart failure. ${ }^{1-3}$ These beneficial effects are thought to result from favourable left ventricular reverse remodelling, which may occur following CRT. Since adverse remodelling (ventricular dilatation), which is a consequence of worsening congestive heart failure, may be associated with increased likelihood of ventricular arrhythmias, CRT alone might be expected to decrease the incidence of ventricular arrhythmias and need for implantable cardioverter defibrillator (ICD) therapy. Whether CRT alone is associated with decreased ventricular arrhythmias or risk of sudden cardiac death is unclear since previous studies have yielded conflicting results. ${ }^{1}{ }^{2} 4-8$
Since advanced or worsening heart failure may increase the risk of ventricular arrhythmias and appropriate ICD therapies, patients with ICDs and worsening heart failure represent an especially high-risk group who may suffer significant morbidity due to frequent ICD therapies. ${ }^{9}$ Since CRT may promote favourable ventricular remodelling and reduce the severity of heart failure, use of CRT might provide an additional therapeutic option to decrease the frequency of appropriate ICD therapies in patients at risk. To address the impact of CRT on the occurrence of ventricular arrhythmias we analysed the frequency of ventricular arrhythmias in the absence and presence of CRT.

\section{PATIENTS AND METHODS}

The protocol was reviewed and approved by the Mayo Clinic Institutional Review Board.

\section{Patients}

All consecutive patients who underwent upgrade from a single or dual chamber ICD to CRTdefibrillator at Mayo Clinic between January 1999 and November 2005 who had $\geqslant 1$ month of followup prior to and following device upgrade were included. Clinical and follow-up data were prospectively entered into a centralised clinical record that includes all clinical evaluations, ICD interrogations and electrograms, echocardiographic data, digital echocardiographic images, operative notes, and digital radiographic records including chest $x$ rays, at Mayo Clinic and affiliated hospitals, and retrospectively analysed. Left ventricular dimensions and ejection fraction prior to and following CRT were measured by echocardiogram in all patients.

\section{CRT-defibrillator upgrade}

All patients were considered eligible for CRT based on accepted clinical criteria. ${ }^{10}$ In all cases, left ventricular pacing was initially attempted via the coronary sinus. If a coronary sinus lead could not be placed, an epicardial left ventricular lead was placed via a thoracotomy. The choice of device, coronary sinus lead, and decision to abandon coronary sinus lead placement were at the discretion of the operator. All patients were observed for $24 \mathrm{~h}$ after upgrade, and coronary sinus lead location and thresholds confirmed with a chest $x$ ray and device interrogation. 
Appropriate device function and pacing thresholds were assessed at $24 \mathrm{~h}$ and at each follow-up. Device reprogramming or lead revision, if necessary, was performed.

\section{Ventricular arrhythmias}

Ventricular arrhythmias were classified as non-sustained (terminated spontaneously), sustained (requiring antitachycardia pacing or shock therapy, excluding ventricular fibrillation), or ventricular fibrillation (cycle length $<230 \mathrm{~ms}$ ). Frequency of ventricular arrhythmias was determined by calculating the mean number of episodes per patient per month. All ventricular arrhythmia events were retrieved at the time of device interrogation during follow-up and entered into a centralised database. Each episode of ventricular arrhythmia was reviewed and classified as non-sustained, sustained ventricular tachycardia or ventricular fibrillation according to our definitions. The frequency (mean number of ventricular arrhythmia episodes per patient per month) of ventricular arrhythmias and appropriate ICD therapies was determined. Since all patients according to the study design underwent upgrade from an ICD to CRT device, episode data from the ICD were obtained retrospectively (although from the same centralised database).

\section{Comparison of frequency of ventricular arrhythmias}

All patients in the study underwent upgrade from an ICD to CRT device, such that each patient served as his or her own control for comparison of frequency of ventricular arrhythmias prior to and following CRT. However, to account for differences in the frequency of ventricular arrhythmias that may have resulted from progression of congestive heart failure or left ventricular dysfunction, we also compared our study group with a cohort of control subjects matched for age, gender, ejection fraction, and ICD device, who had not undergone upgrade of an ICD to a CRT device. To ensure an equivalent length of follow-up to our study patients, we also matched the implant date of the control subjects to the initial ICD implant date of the study patients.

\section{CRT-defibrillator follow-up}

All CRT devices were interrogated at $24 \mathrm{~h}$ and once again at 1 month after implantation, and subsequently every 3 months thereafter. Stored electrograms were obtained during each device interrogation, entered into a centralised database and reviewed and classified according to the same definitions.

\section{Statistical analysis}

Differences in the frequency of ventricular arrhythmias (mean number of episodes per patient per month) prior to and following CRT were compared using a Wilcoxon signed rank test. A p value of $<0.05$ was considered significant. All-cause mortality or need for heart transplantation following CRT was determined (Kaplan-Meier estimates).

\section{RESULTS}

\section{Baseline characteristics}

A total of 53 patients underwent upgrade from ICD to CRTdefibrillator between January 1999 and November 2005 and were included. Baseline characteristics are summarised in table 1. There were 46 men (88\%) and six women (12\%). Mean age was 70 (SD 10) years. Underlying cardiac disease was ischaemic in 41 patients (79\%) and non-ischaemic in 11 patients (21\%). All patients were New York Heart Association functional class IIIII at the time of device upgrade. Initial ICD placement was for secondary prevention of sudden cardiac death in 50 patients $(96 \%)$ and primary prevention in two patients (4\%). Indication for ICD implant in the secondary prevention patients was sustained ventricular arrhythmias or cardiac arrest in 33 patients (63\%), and non-sustained ventricular arrhythmias in association with syncope and inducible ventricular arrhythmias (sustained monomorphic ventricular tachycardia in all cases, induction of non-sustained ventricular arrhythmias ( $\leqslant 30$ beats) or polymorphic ventricular tachycardia was not considered an indication for ICD implantation) at electrophysiology study in 17 patients (33\%). Atrial fibrillation was present in 33 patients (63\%).

Left ventricular end-diastolic and end-systolic dimensions were 68.11 (SD 9.66) $\mathrm{mm}$ and 60.45 (SD 10.50) mm, respectively, and mean ejection fraction was $22 \%$ (SD $8 \%$ ) prior to CRT.

Medications prior to upgrade to a CRT device are summarised in table 2. All patients were on maximal tolerated medical therapy for congestive heart failure. Thirty-six patients (69\%) were taking ACE inhibitors or angiotensin receptor blockers, 35 (67\%) were taking $\beta$ blockers, and 33 (63\%) were on digoxin. A total of 24 patients $(46 \%)$ were on antiarrhythmic drug therapy, with 20 (38\%) on Amiodarone, five (10\%) on Mexilitene, and one $(2 \%)$ on Sotalol. Non-antiarrhythmic cardiac medications are shown in table 2.

\section{CRT device upgrade}

All patients met accepted clinical criteria for upgrade to a CRT device. In 12 patients (23\%) who also met criteria for CRT, upgrade occurred at the time of planned device generator change. No patients were upgraded specifically for the purpose of reducing ventricular arrhythmia burden. Initial implantation success and need for coronary sinus lead revisions are shown in table 3. A coronary sinus lead was successfully placed in 49 patients (94\%). In three patients (6\%), an epicardial left ventricular lead was required. In one of these patients, the epicardial lead failed and a coronary sinus lead was subsequently placed successfully. The epicardial left ventricular lead remained stable in the other two patients. Mean fluoroscopy time was 41.57 (SD 30.26) minutes, which was not significantly different when compared with 46.30 (SD 28.97) minutes in our entire CRT implant population $(\mathrm{p}=0.30)$.

Acute lead revision $(<24 \mathrm{~h})$ was required in three patients; this was for increased pacing thresholds in two, and macrodislodgement of the coronary sinus lead in one patient. Chronic

Table 1 Baseline characteristics

\begin{tabular}{lcc}
\hline Age (mean (SD)) & $70(10)$ years \\
Gender & \multicolumn{2}{l}{} \\
$\quad$ Male & 46 & $(88 \%)$ \\
$\quad$ Female & 6 & $(12 \%)$ \\
Ischaemic Cardiomyopathy & 41 & $(79 \%)$ \\
Non-ischaemic Cardiomyopathy & 11 & $(21 \%)$ \\
ICD implant indication & & \\
$\quad$ Primary prevention & 2 & $(4 \%)$ \\
Secondary prevention & 50 & $(96 \%)$ \\
$\quad$ Ventricular arrhythmia & 33 & $(33 \%)$ \\
$\quad$ Syncope* & 17 & \\
Ejection Fraction (mean (SD)) & $22 \%(8 \%)$ & \\
LVEDD (mean (SD)) & $68.11(9.66) \mathrm{mm}$ & \\
LVESD (mean (SD)) & $60.45(10.50) \mathrm{mm}$ &
\end{tabular}

*inducible ventricular arrhythmia on invasive electrophysiology study. LVEDD, left ventricular end diastolic dimension; LVESD, left ventricular end systolic dimension; SD, standard deviation. 
Table 2 Medications

\begin{tabular}{lrrl}
\hline & $\begin{array}{l}\text { Prior to } \\
\text { CRT (\%) }\end{array}$ & $\begin{array}{l}\text { Following } \\
\text { CRT (\%) }\end{array}$ & p Value \\
\hline Diuretics & $37(71)$ & $34(65)$ & 0.53 \\
ACE inhibitors/Angiotensin & $36(69)$ & $31(60)$ & 0.31 \\
receptor blockers & & & \\
$\begin{array}{l}\text { p-blockers } \\
\text { Digoxin }\end{array}$ & $35(67)$ & $38(73)$ & 0.52 \\
Long-acting Nitrates & $33(63)$ & $36(69)$ & 0.53 \\
Calcium channel blockers & $15(29)$ & $14(27)$ & 0.83 \\
Amiodarone & $2(4)$ & $1(2)$ & 0.56 \\
Mexilitene & $20(38)$ & $24(46)$ & 0.42 \\
Sotalol & $5(10)$ & $5(10)$ & 1.00 \\
\hline
\end{tabular}

CRT, cardiac resynchronisation therapy.

lead revision ( $>24 \mathrm{~h}$ ) was required in two patients at 1 and 19 months following device upgrade. In one of these, right atrial capture was present due to dislodgement of the left ventricular lead into the right atrium. The other had increased pacing thresholds requiring multiple lead revisions. In the remaining 45 patients, the coronary sinus lead remained stable over a mean follow-up time of 14 (SD 12.75) months without the need for lead revision.

\section{Following CRT}

Patient characteristics following CRT are summarised in table 3. Mean ejection fraction increased to $27 \%$ (SD 11\%), an increase of $5 \%(\mathrm{SD} 9 \%)(\mathrm{p}<0.01)$ from baseline. No significant change was observed in left ventricular end diastolic and end systolic dimensions (+0.12 (SD 5.83) mm, p $=0.64$ and -0.60 (SD 5.34) $\mathrm{mm}, \mathrm{p}=0.90$, respectively).

Medication use prior to and following upgrade to a CRTdefibrillator is summarised in table 2. Although use of Digoxin and $\beta$-blockers did increase following upgrade (33 versus 36 patients, $p=0.53,33$ versus 38 patients, $p=0.52$, respectively), while use of ACE inhibitors and angiotensin receptor blockers decreased (36 versus 31 patients, $p=0.31$ ), these differences were not significant. Use of antiarrhythmic drugs was also unchanged following CRT-defibrillator upgrade.

\section{Survival}

Survival of patients following upgrade to CRT device was $88 \%$, $77 \%, 66 \%, 61 \%$ at $6,12,24$, and 36 months, respectively.

\section{Frequency of ventricular arrhythmias}

The frequency of ventricular arrhythmias and appropriate ICD therapies were determined 38.40 (SD 33.02) and 15.63 (SD 11.49) months prior to and following CRT, respectively.

\section{Sustained ventricular arrhythmias}

The frequency of sustained ventricular arrhythmias (requiring either antitachycardia pacing or shock therapy, excluding ventricular fibrillation) was 0.07 (SD 0.17 ) prior to and 0.16 (SD 0.52) following CRT (mean per patient per month) and was not significantly different ( $p=0.70$; fig 2 ).

\section{Ventricular fibrillation}

The frequency of ventricular fibrillation was 0.05 (SD 0.12) prior to and 0.25 (SD 1.40) following CRT (mean per patient per month) and was also not significantly different ( $p=0.12$; fig 3 ).
Table 3 Implantation and follow-up

\begin{tabular}{lll}
\hline & No. of patients & $\%$ \\
\hline Coronary sinus lead implantation success & 49 & 94 \\
Acute lead revision ( $\leqslant 24$ h) & 3 & 6 \\
$\quad$ Increased pacing thresholds & 2 & 4 \\
$\quad$ Macrodislodgement & 1 & 2 \\
Chronic lead revision (>24 h) & 2 & 4 \\
$\quad$ Extra-cardiac stimulation* & 1 & 2 \\
$\quad$ Increased pacing thresholds & 1 & 2 \\
$\quad$ Multiple lead revisions & 1 & 2 \\
Fluoroscopy time (mean (SD)) (minutes) & $41.57(30.26)$ & \\
Ejection Fraction (mean (SD)) & $27 \%(11 \%)$ & \\
LVEDD (mean (SD)) & $68.23(10.63) \mathrm{mm}$ & \\
LVESD (mean (SD)) & $60.05(12.13) \mathrm{mm}$ &
\end{tabular}

*Coronary sinus lead stimulated right atrium. CRT, cardiac resynchronisation therapy LVEDD, left ventricular end-diastolic dimension; LVESD, left ventricular end-systolic dimension; SD, standard deviation.

\section{Non-sustained ventricular arrhythmias}

The frequency of non-sustained ventricular arrhythmias was also unchanged after CRT and was 2.38 (SD 9.78) prior to and 58.51 (SD 412.73) (mean per patient per month) following CRT $(p=0.66$; fig 1A). The large standard deviation was due to one patient with $>40000$ asymptomatic episodes of non-sustained ventricular arrhythmias following CRT. After exclusion of that patient from analysis, no significant difference in the frequency of non-sustained ventricular arrhythmias prior to and following CRT was seen (1.08 (SD 2.68) versus 1.28 (SD 3.58), mean per patient per month, prior to and following CRT $(p=0.65$; fig 1B)).

\section{Comparison with control group}

To determine whether the potential beneficial effect of CRT on the frequency of ventricular arrhythmias was offset by worsening congestive heart failure, we identified 52 patients matched for age, gender, ejection fraction, device type, and implant date to serve as a control group. Of these, $34 \mathrm{had} \geqslant 1$ month of follow-up and were used for comparison of frequency of ventricular arrhythmias prior to and following CRT. During a mean follow-up time of 48.47 (SD 32.03) months, the frequency of sustained ventricular arrhythmias, ventricular fibrillation, and non-sustained ventricular arrhythmias was 2.49 (SD 12.69), 0.04 (SD 0.12), and 2.00 (SD 6.46), respectively. No significant differences in the frequency of ventricular arrhythmias prior to or following CRT $(p=0.27,0.73,0.82$ prior to CRT, $p=0.29$, $0.29,0.33$ following CRT) were observed in comparison with the study patients.

\section{DISCUSSION}

The major finding of this study is that, in patients with congestive heart failure and usual indications for CRT, CRT is not associated with a decrease in the frequency of sustained and non-sustained ventricular arrhythmias or appropriate device therapy despite evidence of favourable ventricular remodelling following CRT. We also found that the frequency of ventricular arrhythmias in patients with severe left ventricular dysfunction treated with CRT is not different from patients with similar clinical characteristics who are not receiving CRT. Therefore, use of CRT to decrease the frequency of ventricular arrhythmias or appropriate device therapy is not warranted.

Our findings contrast with those of previous studies that have reported both an increase and a decrease in the frequency of ventricular arrhythmias following CRT.46811-13 The mechanism 
Figure 1 A. Frequency of non-sustained ventricular arrhythmias per patient per month prior to (pre-CRT) and following (post-CRT) upgrade from an ICD to a CRTdefibrillator. Each point represents mean number of events per month in an individual patient. B. Identical plot of frequency of non-sustained ventricular arrhythmias after exclusion of patient with $>40000$ episodes. Mean number of events per month for the entire study group, both pre-CRT and post-CRT, is shown (grey line).
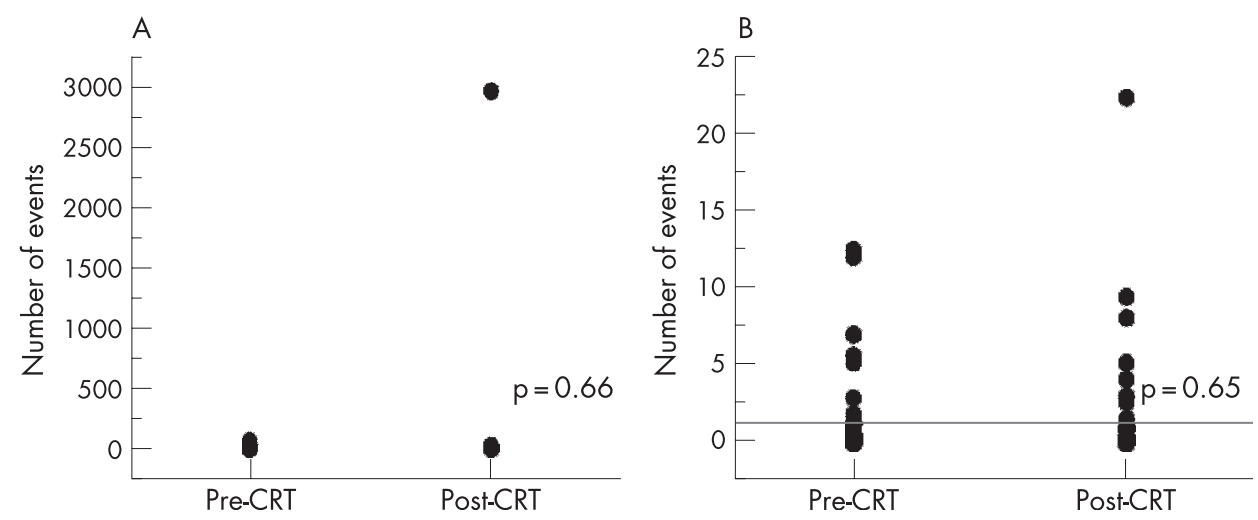

by which CRT is thought to improve outcome is via decreased wall stress due to reversed remodelling. Although we did not observe a significant decrease in left ventricular size, we did observe a similar increase in ejection fraction following CRT to that of two previous studies that found decreased frequency of ventricular arrhythmias following upgrade from an ICD to a CRT-defibrillator, suggesting that our patients had a similar haemodynamic benefit from CRT, yet no change in the frequency of ventricular arrhythmias. ${ }^{4}{ }^{8}$ These studies, however, were limited by small sample size and may have overestimated the effect of CRT on ventricular arrhythmia burden. In contrast, our study represents the largest single-centre series of patients undergoing upgrade from an ICD to a CRT-defibrillator, with the longest follow-up time. Moreover, by comparison of our study patients with a matched control group, we have demonstrated that the frequency of ventricular arrhythmias is similar in patients with and without CRT devices, which further supports the notion that CRT does not impact on the occurrence of ventricular arrhythmias.

It has been reported that CRT may also have a proarrhythmic potential. Although several mechanisms may underlie this, animal studies using epicardial pacing via the coronary sinus have demonstrated prolongation of the QT interval and torsades des pointes. ${ }^{12}$ A pro-arrhythmic effect of CRT has also been reported in humans. ${ }^{11} 1314$ Although we did in fact observe an increase in the number of ventricular arrhythmia episodes after CRT, this difference was not statistically significant.

Our data demonstrating a neutral effect of CRT on ventricular arrhythmias is consistent with the findings of the

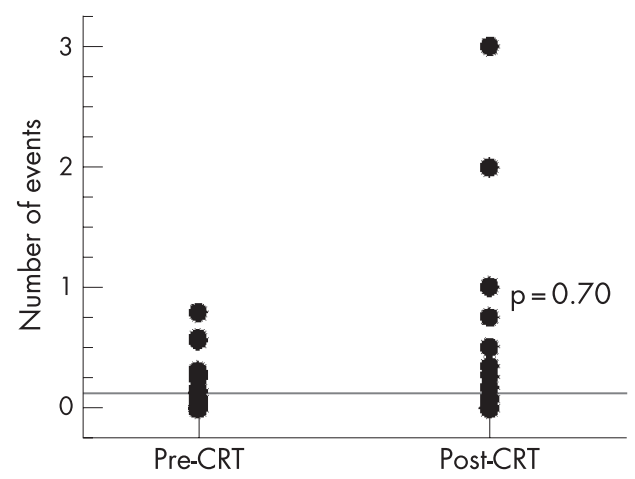

Figure 2 Frequency of sustained ventricular arrhythmias per patient per month prior to (pre-CRT) and following (post-CRT) upgrade from an ICD to a CRT-defibrillator. Each point represents mean number of events per month in an individual patient. Mean number of events per month for the entire study group, both pre-CRT and post-CRT, is shown (grey line).
CONTAK-CD and Multisite InSync Randomized Clinical Evaluation-ICD (MIRACLE-ICD) trials, which also found no effect of CRT on ventricular arrhythmias or appropriate ICD therapies. ${ }^{157}$ The importance of our findings is that all data are derived from a single-centre experience with uniform and complete follow-up, thereby avoiding possible confounding due to differences in device follow-up and programming. The impact of CRT alone on mortality is less clear. A recent metaanalysis including data from the extension phase of Cardiac Resynchronization-Heart Failure (CARE-HF) provided evidence against a benefit of CRT alone on risk of sudden cardiac death. ${ }^{15} 16$ These findings are consistent with ours and suggest that the mortality benefit of CRT is not related to a reduction in the frequency of ventricular arrhythmias but is more likely related to improved haemodynamic status as a consequence of ventricular reverse remodelling.

In conclusion, in patients with usual indications for CRT undergoing upgrade from ICD therapy, CRT alone is not associated with a reduction in the frequency of ventricular arrhythmias or ventricular fibrillation. Therefore, these data do not support the use of CRT alone as an effective therapy for frequent ventricular arrhythmias and appropriate ICD shocks in patients with worsening heart failure at risk of sudden cardiac death.

\section{STUDY LIMITATIONS}

Due to the retrospective study design, differences in device programming and/or initiation of antiarrhythmic drug therapy occurring as a result of a documented ventricular arrhythmia

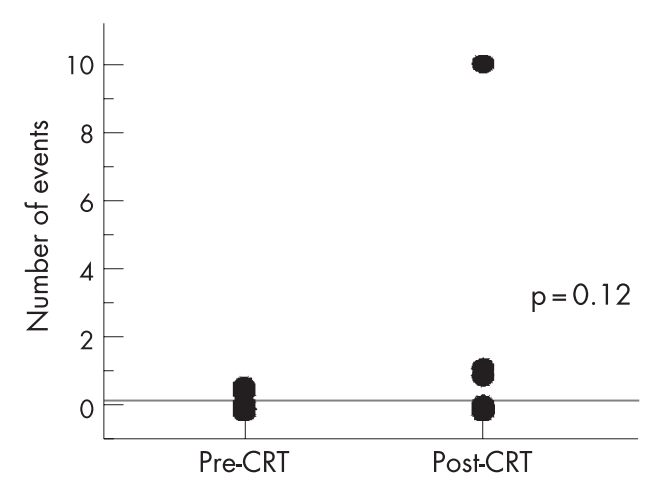

Figure 3 Frequency of ventricular fibrillation per patient per month prior to (pre-CRT) and following (post-CRT) upgrade from an ICD to a CRTdefibrillator. Each point represents mean number of events per month in an individual patient. Mean number of events per month for the entire study group, both pre-CRT and post-CRT, is shown (grey line). 
could not be accounted for. However, use of antiarrhythmic drug therapy was unchanged overall during the study period. In addition, due to the relatively small sample size, differences in outcome and occurrence of ventricular arrhythmias could not be determined in "responders" versus "non-responders" to CRT.

\section{CONCLUSIONS}

Based upon our findings, CRT does not decrease the frequency of ventricular arrhythmias, and therefore upgrade of an ICD to a device capable of CRT to decrease the frequency of appropriate ICD therapies or to reduce symptoms from ventricular arrhythmias is unlikely to be of benefit.

Funding: This study was not supported by any financial grants.

Competing interests: Dr Hayes has the following financial disclosures: Educational speaking; Medtronic, Boston Scientific, St Jude Medical, Sorin/ELA, Sponsored research; Medtronic, Boston Scientific, St Jude Medical, Steering committee: Medtronic, Advisory board; Boston Scientific, Sorin/ELA, St Jude Medical.

\section{REFERENCES}

1. Young J, Abraham W, Smith A, et al, for Multisite InSync Randomization Clinical Evaluation (MIRACLE-ICD) investigators. Combined cardiac resynchronization and implantable cardioverter defibrillation in advanced chronic heart failure: the MIRACLE ICD trial. JAMA 2003;289:2685-94.

2. Bristow M, Saxon L, Boehmer J, et al, for the Comparison of Medical Therapy Pacing, and Defibrillation in Heart Failure (COMPANION) Investigators. Cardiacresynchronization therapy with or without an implantable cardioverter defibrillator in advanced chronic heart failure. N Engl J Med 2004;350:2140-50.

3. Cleland J, Daubert J, Erdmann E, et al, for the Cardiac Resynchronization-Heart Failure (CARE-HF) study investigators. The effect of cardiac resynchronization on morbidity and mortality in heart failure. N Engl J Med 2005;352:1539-49.

4. Ermis C, Seutter R, Zhu A, et al. Impact of upgrade to cardiac resynchronization therapy on ventricular arrhythmia frequency in patients with implantable cardioverterdefibrillators. J Am Coll Cardiol 2005;46:2258-63.
5. McSwain R, Schwartz R, DeLurgio D, et al. The impact of cardiac resynchronization therapy on ventricular tachycardia/fibrillation: an analysis from the combined ContakCD and InSync-ICD studies. J Cardiovasc Electrophysiol 2005;16:1168-71.

6. Higgins S, Yong $P$, Scheck D, et al. Biventricular pacing diminishes the need for implantable cardioverter defibrillator therapy. J Am Coll Cardiol 2000;36:824-7.

7. Higgins S, Hummell J, Niazi I, et al. Cardiac resynchronization therapy for the treatment of heart failure in patients with intraventricular conduction delay and malignant ventricular tachyarrhythmias. J Am Coll Cardiol 2003;42:1454-9.

8. Kies $\mathbf{P}$, Bax J, Molhoek $\mathrm{S}$, et al. Effect of left ventricular remodeling after cardiac resynchronization therapy on frequency of ventricular arrhythmias. Am J Cardiol 2004;94:130-2.

9. Singh JP, Hall WJ, McNitt $S$, et al. Factors influencing appropriate firing of the implanted defibrillator for ventricular tachycardia/fibrillation: findings from the Multicenter Automatic Defibrillator Implantation Trial II (MADIT-II). J Am Coll Cardiol 2005; 46:1712-20.

10. Hunt S. ACC/AHA 2005 Guideline update for the diagnosis and management of chronic heart failure in the adult: a report of the American College of Cardiology/ American Heart Association Task Force on Practice Guidelines (Writing Committee to Update the 2001 Guidelines for the Evaluation and Management of Heart Failure). J Am Coll Cardiol 2005;46:e1-82.

11. Guerra J, Wu J, Meller J, et al. Increase in ventricular tachycardia frequency after biventricular implantable cardioverter defibrillator upgrade. J Cardiovasc Electrophysiol 2003;14:1245-7.

12. Fish J, Di Diego J, Nesterenko V, et al. Epicardial activation of left ventricular wall prolongs QT interval and transmural dispersion of repolarization: implications for biventricular pacing. Circulation 2004;109:2136-42.

13. DiCori A, Bongiorni M, Arena G, et al. New-onset ventricular tachycardia after cardiac resynchronization therapy. J Interv Card Electrophysiol 2005; 12:231-5

14. Medina-Ravell V, Lankipalli R, Yan G, et al. Effect of epicardial or biventricular pacing to prolong OT interval and increase transmural dispersion of repolarization: does resynchronization therapy pose a risk for patients predisposed to long QT or torsade de pointes? Circulation 2003;107:740-6.

15. Cleland JGF, Daubert J-C, Erdmann E, et al. Longer-term effects of cardiac resynchronization therapy on mortality in heart failure [the CArdiac REsynchronizationHeart Failure (CARE-HF) trial extension phase]. Eur Heart J 2006;27:1928-32.

16. Rivero-Ayerza M, Theuns DAMJ, Garcia-Garcia HM, et al. Effects of cardiac resynchronization therapy on overall mortality and mode of death: a meta-analysis of randomized controlled trials. Eur Heart J 2006;27:2682-8.

\section{Submit an eletter, and join the debate}

eLetters are a fast and convenient way to register your opinion on topical and contentious medical issues. You can find the "submit a response" link alongside the abstract, full text and PDF versions of all our articles. We aim to publish swiftly, and your comments will be emailed directly to the author of the original article to allow them to respond. eLetters are a great way of participating in important clinical debates, so make sure your voice is heard. 